Trauma Berufskrankh 2013 · 15[Suppl 1]:17-24 DOI 10.1007/s10039-012-1904-7

Online publiziert: 22. August 2012

(c) Springer-Verlag Berlin Heidelberg 2012

\section{G. Jensen · C. Katthagen · H. Lill · C. Voigt}

Abteilung für Unfall- und Wiederherstellungschirurgie, Diakoniekrankenhaus Friederikenstift gGmbH

Hannover, Abteilung des Berufsgenossenschaftlichen Unfallkrankenhauses Hamburg, Hannover

\section{Hintergrund}

Verletzungen des Schultereckgelenks (ACG: Akromioklavikulargelenk) gehören aufgrund ihrer Häufigkeit zum chirurgischen Alltag. Wie für kaum eine andere Verletzung steht eine Vielzahl von operativen Strategien zur Verfügung, was die bei der Versorgung vorhandenen Schwierigkeiten verdeutlicht.

Neben den konventionellen Techniken konnten sich in den letzten Jahren arthroskopisch gestützte neue Therapieansätze etablieren. Diesbezüglich stellen sich folgende Fragen

- Was sind die neuen Erkenntnisse aus diesen Verfahren?

- Gibt es neue Therapiekonzepte bei chronischen ACG-Instabilitäten?

\section{Epidemiologie und Pathophysiologie von Schultereckgelenkverletzungen}

Verletzungen des Schultereckgelenks sind relativ häufig und machen etwa $9 \%$ aller Läsionen des Schultergürtels aus. Überwiegend sind sportlich aktive Männer (Verhältnis Männer:Frauen 5:1) zwischen dem 20. und 40. Lebensjahr betroffen [17].

Pathophysiologisch ursächlich ist oft ein direkter Sturz auf die betroffene posterosuperiore Schulter mit adduziertem Arm [6], wie er überwiegend im Rahmen von Stürzen bei Kontaktsportarten, alpinen Unfällen oder Zweiradunfällen auftritt.

\section{Verletzungstypen \\ und Therapieoptionen}

Geringgradige Verletzungen des ACG (Typ Rockwood I und II) werden in der Akutphase konservativ behandelt, wohingegen die Therapie der ACG-Sprengungen Typ III nach Rockwood nach wie vor kontrovers diskutiert wird. Aktuell existiert kein Konsens darüber, ob akute Verletzungen dieser Art operativ oder konservativ versorgt werden sollten. Einigkeit scheint dahingehend zu bestehen, dass Verletzungen vom Typ Rockwood IV-VI einer operativen Behandlung zugeführt werden sollten [4].

In der Literatur findet sich eine Vielzahl verschiedener Techniken zur Versorgung akuter Instabilitäten mit einer fast ebenso großen Anzahl an Implantaten [17]. Implantatassoziierte Komplikationen sind häufig, wie beispielsweise KirschnerDraht-Migration bei temporären Transfixationen, Schraubenbruch bei der Bosworth-Schraube oder subakromiale Irritationen und Stressfrakturen des Akromions bei der Hakenplattentechnik [5].

Eine andere Entität der Erkrankung ist die chronische Instabilität. Im Gegensatz zur akuten Verletzung, bei welcher durch die operativen Verfahren eine Reposition und temporäre Retention des ACG herbeigeführt werden sollen, damit die kapsuloligamentären Strukturen ein narbiges Regenerat bilden, ist diese Regenerationsfähigkeit bei chronischen Verläufen nicht mehr vorhanden. Die Bänder müssen durch ein biologisches Verfahren, wie eine Transposition des korakoakromialen Bandes nach Weaver-Dunn oder eine Plastik der korakoklavikularen (CC) Bänder durch eine autologe Sehne ersetzt werden.

Der Zeitraum, ab wann eine chronische Instabilität vorliegt, ist nicht klar definiert. Bei einer verspäteten Versorgung ist mit signifikant schlechteren Resultaten zu rechnen [24]. Die Zeitspanne zwischen Trauma und Operation sollte, falls ein operatives Vorgehen indiziert ist, möglichst kurz (<3 Wochen) sein [19]. Andernfalls sollte nach den Therapieempfehlungen bei chronischer Instabilität vorgegangen werden.

\section{Anatomie und Biomechanik}

Das Schultereckgelenk ist ein diarthrodiales Gelenk, das über 3 Freiheitsgrade verfügt. Neben der Rotation handelt es sich v. a. um die Translation in a.-p. sowie in inferosuperiorer Richtung. Das Gelenk dient als Drehpunkt für Protraktionsund Retraktionsbewegungen der Skapula am Rumpf und über den korakoklavikularen Ligamentkomplex als Aufhängung des Schultergürtels [17].

Das ACG verfügt mit dem intraartikulärem Diskus über eine meniskusähnliche Struktur. Diese unterliegt rasch degenerativen Veränderungen bis zum praktischen Funktionsverlust ab der 4. Lebensdekade [22], sodass die Rolle dieser Struktur, v. a. beim Trauma, zu vernachlässigen ist [17].

Das ACG besitzt statische und dynamische Stabilisatoren. Erstere sind die 
Tab. 1 Übersicht der verletzten Strukturen sowie Änderungen des CC-Abstands. (Nach [23])

\begin{tabular}{|c|c|c|c|c|c|c|}
\hline Rockwood-Typ & I & II & III & Typ IV & Typ V & VI \\
\hline $\mathrm{ACL}$ & Gezerrt & Rupturiert & Rupturiert & Rupturiert & Rupturiert & Rupturiert \\
\hline ACG & Intakt & $\begin{array}{l}\text { Gelenkspalt weiter, } \\
\text { leichter Vertikalversatz }\end{array}$ & Disloziert & $\begin{array}{l}\text { Klavikula nach posterior } \\
\text { in M. trapezius disloziert }\end{array}$ & Grob disloziert & Disloziert \\
\hline $\mathrm{CCL}$ & Intakt & Gezerrt & Rupturiert & Rupturiert & Rupturiert & Rupturiert \\
\hline $\begin{array}{l}\text { Deltoideo- } \\
\text { trapezoidale } \\
\text { Faszie }\end{array}$ & Intakt & Intakt & $\begin{array}{l}\text { Meistens von distalen } \\
\text { Ende der Klavikula } \\
\text { gelöst }\end{array}$ & $\begin{array}{l}\text { Vom distalen Ende } \\
\text { abgelöst }\end{array}$ & $\begin{array}{l}\text { Von distaler } \\
\text { Hälfte der Klavi- } \\
\text { kula abgelöst }\end{array}$ & $\begin{array}{l}\text { Abgelöst vom distalen } \\
\text { Ende der Klavikula }\end{array}$ \\
\hline CC-Abstand $^{\mathrm{a}}$ & $\begin{array}{l}\text { Unver- } \\
\text { ändert }\end{array}$ & 0 bis $+25 \%$ erweitert & $\begin{array}{l}+25 \text { bis }+100 \% \\
\text { erweitert }\end{array}$ & $\begin{array}{l}\text { Kann unverändert } \\
\text { erscheinen }\end{array}$ & $\begin{array}{l}+101 \text { bis } \\
+300 \%\end{array}$ & $\begin{array}{l}\text { Klavikula unter Processus } \\
\text { coracoideus und hinter } \\
\text { "conjoint tendon“ luxiert } \\
\text { (extrem selten) }\end{array}$ \\
\hline
\end{tabular}

AC-Ligamente (ACL), welche die Gelenkkapsel verstärken, sowie die korakoklavikularen Ligamente (CCL, Lig. trapezoideum und conoideum) und das korakoakromiale Band. Die dynamischen Stabilisatoren bestehen aus dem M. deltoideus, dem M. trapezius und dem M. serratus anterior. Faserzüge der deltatrapezoidalen Faszie verstärken die superioren AC-Bänder.

Die CCL haben 2 Hauptfunktionen:

- Sie führen durch die Aufhängung der Skapula an der Klavikula den skapulohumeralen Bewegungsablauf.

- Sie sind der Hauptstabilisator des ACG in der Vertikalebene [7].

Die ACL dagegen sind der Hauptstabilisator des ACG in der a.-p. Ebene, hier v. a. nach posterior (superiores ACL 56\% und posteriores ACL 25\%, [15]).

Der skapulohumerale Bewegungsablauf sowie die Rolle der einzelnen Stabilisatoren sind insgesamt komplex. Alle Strukturen sollten im Rahmen therapeutischer Interventionen mit adressiert werden, um ein optimales Ausheilungsergebnis zu erreichen [7].

\section{Klassifikation der ACG-Sprengung}

Im klinischen Alltag etablierte sich die Klassifikation nach Rockwood [23] und löste weitgehend die Einteilung nach Tossy et al. [32] ab, da sie insbesondere bei den höhergradigen Verletzungen differenzierter ist (• Tab. 1; [23]).

Eine Schwäche der Klassifikation nach Rockwood [23] ist, dass sie sich zur Abschätzung des Verletzungsausmaßes an der radiologischen Veränderung des CCAbstands im Vergleich zur Gegenseite orientiert. Horizontale Instabilitäten finden hierbei bis auf die statische posteriore Instabilität mit der Dislokation nach dorsal in den M. trapezius beim Typ Rockwood IV keine Berücksichtigung. Gerade bei der Typ-III- und -V-Verletzung liegen jedoch häufig auch dynamische horizontale Instabilitäten vor. Patienten mit verbliebener horizontaler Instabilität zeigen ein signifikant schlechteres klinisches Outcome [12, 26]. Hedtmann et al. [11] hoben bereits 1998 die Bedeutung der horizontalen Instabilität bei der Entstehung von chronisch symptomatischen Instabilitäten am ACG hervor. Sinnvoll wäre hier, v. a. auch für eine differenzierte Beratung und Therapieempfehlung, eine Erweiterung des Typs III nach Rockwood in Verletzungen ohne (Rockwood IIIa) und mit (Rockwood IIIb) relevanter horizontaler Instabilität.

\section{Klinik und Diagnostik}

Die Anamnese sowie der Unfallmechanismus sind oft richtungweisend.

Inspektorisch imponiert bei der akuten höhergradigen ACG-Sprengung ein Akromiontiefstand der betroffenen Seite, häufig ist an der posterosuperioren Schulter eine begleitende Prellmarke ersichtlich.

Palpatorisch besteht eine Druckdolenz über dem ACG. Es zeigt sich das typische sog. Klaviertastenphänomen als Zeichen der vertikalen Instabilität ab RockwoodIII-Verletzungen. Das Cross-Body-Zeichen ist meist positiv.
Wichtig ist auch die klinische Funktionsprüfung einer horizontalen Instabilität mit pathologischem Shift der lateralen Klavikula v. a. nach posterior. Die Beweglichkeit ist in der Akutphase oft schmerzbedingt höhergradig eingeschränkt. Differenzierte Funktionstestungen möglicher Begleitpathologien an der Rotatorenmanschette oder der Bizepssehne/dem SLAPKomplex (SLAP: „superior labrum anterior to posterior") sind häufig schmerzbedingt nur eingeschränkt durchführbar, jedoch sollte stets eine komplette Untersuchung des gesamten Schultergürtels erfolgen.

Bei der chronisch-symptomatischen ACG-Instabilität finden sich klinisch die oben angeführten Instabilitätszeichen. Die Beweglichkeit ist meist wieder frei. Häufig besteht jedoch ein endgradiger sog. schmerzhafter Bogen („painful arc"). Beim Cross-Body-Manöver ist oft ein Höhertreten des lateralen Klavikulaendes zu beobachten, wenn dieses auf das Akromion aufgeschoben wird. Häufig besteht dann ein schmerzhaftes spinoklavikulares Impingement [17]. Bei chronischen Instabilitäten findet sich ein gestörter skapulothorakaler Bewegungsrhythmus. Dieser ist durch die gestörte Aufhängung und den fehlenden festen Drehpunkt der Skapula am Rumpf bedingt, was neben dem Tiefertreten der Skapula einen Shift nach anterolateral zur Folge hat. Nicht selten führt dies bis zu einem SICK-SkapulaSyndrom (SICK: „scapular malposition, inferior medial border prominence, coracoid pain and malposition, and dyskinesis of scapular movement"; [9]). Durch die Fehlposition der Skapula wird ein Kontakt des Processus coracoideus und des M. 
pectoralis minor mit den Plexus brachialis begünstigt. Hieraus können Nervenirritationssyndrome analog eines ThoracicOutlet-Syndroms resultieren [11].

Zur Instabilitätsdiagnostik am ACG gehört routinemäßig folgende Röntgendiagnostik (• Abb. 1):

- bilaterale Zielaufnahmen des ACG als Stressaufnahmen mit $10 \mathrm{~kg}$ Belastung zur Beurteilung der vertikalen Instabilität,

- eine axiale Aufnahme zur Beurteilung der statischen Dislokation nach posterior,

- die bilaterale Aufnahme nach Alexander [1] als Modifikation einer Y-Aufnahme im Sinne einer Stressaufnahme zur Beurteilung der dynamischen Instabilität nach posterior.

Exakte Evaluationen, ab wann eine horizontale Instabilität bei der AlexanderAufnahme klinisch relevant ist, gibt es bisher nicht.

Tauber et al. [29] etablierten ein dynamisches Röntgenverfahren mit der Bestimmung des glenoakromioklavikularen Winkels aus 2 axialen Röntgenaufnahmen:

1. $90^{\circ} \mathrm{Abduktion}$ und neutrale Flexion;

2. $90^{\circ}$ Abduktion und $60^{\circ}$ Flexion.

$\mathrm{Ab}$ einer Zunahme des Winkels $>12,3^{\circ}$ liegt gemäß Tauber et al. [29] eine relevante horizontale Instabilität vor.

MRT-Untersuchungen (MRT: Magnetresonanztomographie) können zur Detektion von relevanten Begleitpathologien v. a. bei chronischen ACG-Läsionen hilfreich sein, sind jedoch nicht routinemäßig in allen Fällen zu erstellen.

Die CT (Computertomographie) bleibt speziellen Fragestellungen vorbehalten.

\section{Therapie}

\section{Akute Rockwood-I- und -II-Läsionen}

Sie werden konservativ und symptombezogen behandelt. Neben analgetischer Medikation sowie lokal lindernden Maßnahmen (Eisanwendung) ist eine kurzfristige Ruhigstellung in einer Schulter-ArmSchlinge möglich [17].

Generell werden im Rahmen einer konservativen Therapie klinisch gute Re-

Trauma Berufskrankh 2013 · 15[Suppl 1]:17-24 DOI 10.1007/s10039-012-1904-7

(c) Springer-Verlag Berlin Heidelberg 2012

\author{
G. Jensen · C. Katthagen · H. Lill · C. Voigt
}

\title{
Aktuelle Versorgungsstrategien bei Schultereckgelenkinstabilitäten
}

\section{Zusammenfassung}

Verletzungen am Schultereckgelenk gehören aufgrund ihrer Häufigkeit zum klinischen Alltag. Rockwood(RW)-I- und -II-Läsionen werden initial konservativ behandelt. Die optimale Therapie einer RW-III-Verletzung wird nach wie vor kontrovers diskutiert. Höhergradige Verletzungen sollten einer operativen Behandlung zugeführt werden. Neueren Erkenntnissen zufolge sollte insbesondere bei der RW-III-Läsion einer horizontalen Komponente der Instabilität Rechnung getragen und diese in die Beratung der Patienten sowie das operative Konzept mit integriert werden. Sowohl bei den akuten als auch den chronischen Instabilitäten konnten sich zunehmend die arthroskopisch gestützten Ver- fahren etablieren. Bei akuten Verletzungen erzielen Fixationen mit Endobuttonsystemen sehr gute Resultate. Bei chronischen Instabilitäten ist eine anatomische Rekonstruktion der ligamentären Strukturen durch ein autologes Sehnentransplantat, welches durch ein Endobuttonsystem augmentiert wird, ebenso eine Option wie eine Transposition des korakoakromialen Ligaments.

\section{Schlüsselwörter}

Schultereckgelenk · Gelenkinstabilität · Arthroskopische Operation · Rekonstruktion der korakoklavikularen Bänder · Horizontale Augmentation

\section{Current treatment strategies for instabilities of the acromioclavicular joint}

\section{Abstract}

Injuries of the acromioclavicular joint are common in clinical daily routine. Rockwood (RW) I and II lesions are treated non-operatively. The ideal treatment for RW III lesions is still controversially discussed. RW IV-VI injuries require operative treatment. New findings emphasize the importance of a horizontal component of instability at the RW III injury, which should be integrated into the medical advice for an operative or non-operative treatment and the operative concept. During recent years arthroscopically-assisted techniques have become established for repair of acute as well as chronic instabilities. In acute lesions very good clinical results can be archived with fixation techniques using endobutton systems. In cases of chronic instability, options include anatomic reconstruction of the ligamentous structures with a tendon autograft augmented by an endobutton system as well as transposition techniques of the coracoacromial ligament.

\section{Keywords}

Acromioclavicular joint · Joint instability · Arthroscopic surgery - Reconstruction of coracoclavicular ligaments $\cdot$ Horizontal augmentation sultate erzielt, nicht selten zeigen sich jedoch im Verlauf degenerative Veränderungen am ACG durch eine verbliebene Restinstabilität sowie Kalzifikationen der CCL [18].

Bei relevanten klinischen Beschwerden kann die laterale Klavikula, jedoch in spärlichem Ausmaß (etwa $5 \mathrm{~mm}$ ), reseziert werden. Dabei sollten das posteriore und das superiore ACL geschont werden, damit es nicht zu einer weiteren Destabilisierung der lateralen Klavikula in horizontaler Ebene kommt [2].

\section{Rockwood-III-Verletzungen - operative vs. konservative Behandlung}

Die Frage, ob eine Rockwood-III-Verletzung operativ oder konservativ versorgt werden sollte, wird nach wie vor kontrovers diskutiert [4]. Es besteht derzeit ein Mangel an höherwertigen randomisierten Studien, um diese Frage gemäß den angewandten Kriterien der evidenzbasierten Medizin adäquat beantworten $\mathrm{zu}$ können [27]. Die bisherigen Vergleichsstudien beziehen sich ausschließlich auf 


\section{Schulterverletzungen}
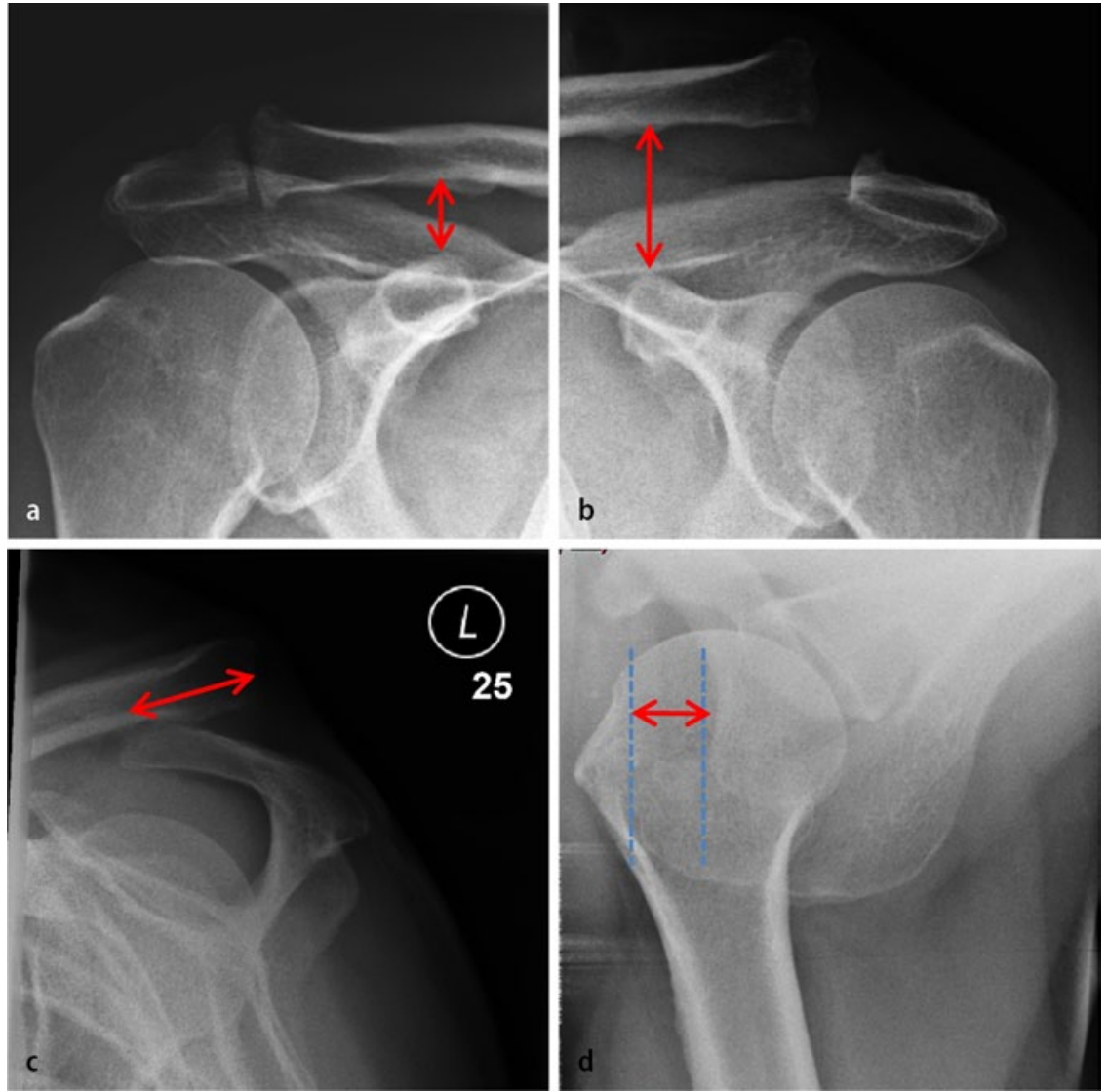

Abb. $1 \Delta$ Röntgendiagnostik: a,b bilaterale ACG-Zielaufnahmen mit je $10 \mathrm{~kg}$ Belastung zur genauen Beurteilung des CC-Abstandes (roter Doppelpfeil) und Festlegung der Rockwood-Klassifikation, c Alexander-Aufnahme zur Beurteilung der horizontalen Instabilitätskomponente (roter Doppelpfeil Aufschieben des lateralen Klavikulaendes auf das Akromion), d axiale Aufnahme zur Beurteilung des Versatzes der lateralen Klavikula gegenüber dem Akromion nach dorsal [Doppelpfeil Strecke zwischen anteriorer Akromionkante (blaue Linie) und Vorderkante der lateralen Klavikula], ACG Akromioklavikulargelenk, CC korakoklavikular

die konventionellen Techniken der ACGStabilisierung wie Kirschner-Draht-Fixierung, PDS-Kordel (PDS: Polydioxansulfat), Hakenplatte oder Bosworth-Schraube. Prospektive randomisierte Vergleichsstudien zu den neuen, arthroskopisch gestützten Techniken fehlen bis dato. Bisher nicht berücksichtig wurde die Tatsache, dass es sich bei der Gruppe der Rockwood-III-Läsionen nicht um eine homogene Gruppe handelt, sondern zwischen Patienten mit und ohne horizontale Instabilität unterschieden werden muss. Die Bedeutung der horizontalen Instabilität bei dieser Art der Verletzung als Prädiktor für ein schlechteres Outcome sollte in der Therapieentscheidung mit berücksichtigt werden $[12,26]$.

Eine differenzierte Beratung ist erforderlich, um gemeinsam mit dem Patienten die für ihn optimale Wahl zu treffen.
Hierbei sollten u. a. das Alter des Patienten, der sportliche Anspruch, der Beruf (Überkopftätigkeit/körperlich schwere Arbeit) und die betroffenen Seite (dominierender Arm) in die Beratung mit einbezogen werden. Ebenfalls wichtig ist die Tatsache, dass ein operatives Vorgehen nach mehreren Monaten bei chronischen Beschwerden aufwändiger ist, da dann ein biologisches Augmentationsverfahren erforderlich wird und mit einem schlechteren klinischen Outcome zu rechnen ist [24].

\section{Operative Versorgung höhergradiger ACG-Sprengungen}

Während die Therapieentscheidung bei der Rockwood-III-Verletzung den oben genannten Kriterien vorbehalten bleibt, besteht Einigkeit darüber, dass höher- gradige ACG-Verletzungen operativ versorgt werden sollten [4].

Neben den konventionellen Techniken, bei denen aktuell meist die Hakenplatte favorisiert wird, konnten sich in den letzten Jahren die arthroskopisch gestützten Verfahren etablieren. Bei der Hakenplatte, die sehr rigide, jedoch nicht anatomisch ist, liegen mittelfristige Ergebnisse mit teils sehr guten klinischen Resultaten vor [14]. Wesentlicher Nachteil dieses Verfahrens ist der zur Implantatentfernung erforderliche Zweiteingriff.

Auch zu den neuen Verfahren wie der Tight-Rope-Technik liegen inzwischen 2-Jahres-Ergebnisse mit sehr guten klinischen Resultaten vor [26].

Im eigenen Patientengut konnten sowohl mit der Hakenplatten- als auch mit der Doppel-Tight-Rope-Technik gute und sehr gute klinische Ergebnisse erzielt werden, wobei das arthroskopisch gestützte Verfahren die konventionelle Technik vollständig ablöste. Prospektiv randomisierte Studien zum Vergleich beider Methoden sind bisher jedoch nicht publiziert.

Neben dem einzeitigen Vorgehen sind die Identifikation und Therapie von relevanten intraartikulären Begleitpathologien wie SLAP-Läsionen oder Verletzungen der Rotatorenmanschette ein Vorteil des arthroskopisch gestützten Verfahrens. Die Häufigkeit intraartikulärer Begleitpathologien wird mit $15-18,5 \%$ angegeben $[21,31]$.

Bei beiden Verfahren kann es im Verlauf zur sekundären Dislokation des ACG kommen $[10,26]$. Gründe hierfür sind eine insuffiziente Narbenbildung im Verlauf der CCL mit einem Repositionsverlust nach Implantatentfernung bei der Hakenplattentechnik oder ein vorzeitiges $\mathrm{Fa}$ denversagen oder eine Migration der klavikularen oder korakoidalen Buttons bei der Tight-Rope-Technik vor dem vollständigen Ausheilen der Bänder [20].

Die Frage, ob eine Einzel- oder eine Doppel-Tight-Rope-Technik zu bevorzugen ist, scheint nicht endgültig geklärt. Bei den meisten Publikationen fand Letztere Anwendung [12, 26, 31]. Patzer et al. [20] verglichen die Einzel- mit der Doppel-Tight-Rope-Technik und fanden keine signifikanten Unterschiede bezüglich des Repositionsergebnisses und der klini- 

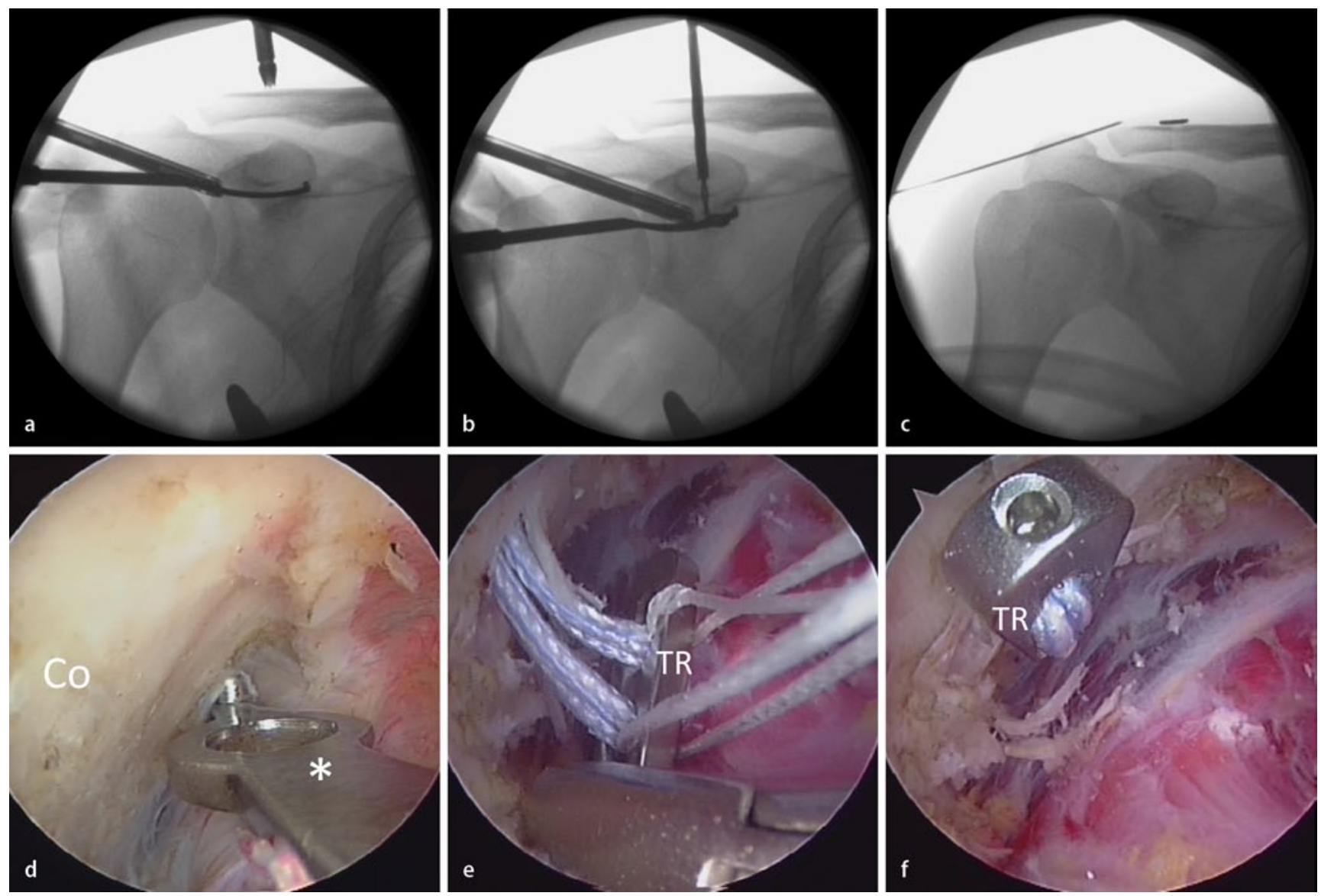

Abb. $2 \triangle$ Operationstechnik bei akuter Akromioklavikalurgelenkinstabilität, a,b bildwandlergestützte Platzierung der transklavikularen transakromialen Bohrung, a Positionierung des Zielbügels, b Überbohrung, $\mathbf{c}$ transakromiale Bohrung für die Horizontalcerclage, d Zielinstrument $\left(^{*}\right)$ an Korakoidunterseite $(C O)$, e Einbringen des Tight-Rope $(T R), \mathbf{f}$ subkorakoidale Position des Fixationsbuttons, weitere Erläuterungen s. Text

schen Resultate bei den Follow-up-Untersuchungen.

Im eigenen Vorgehen wird inzwischen wieder die Einzel-Tight-Rope-Technik favorisiert, die jedoch aufgrund der Relevanz der horizontalen Stabilität um eine zusätzliche horizontale transklavikulare, transakromiale FibreTape ${ }^{\mathrm{m} x}$-Cerclage ergänzt wurde [8].

\section{Operationstechnik bei akuter ACG-Instabilität}

Nach Etablieren des dorsalen Optikportals und des anteroinferioren Arbeitszugangs erfolgen die glenohumerale Inspektion und ggf. die Therapie intraartikulärer Begleitpathologien. Anschließend wird in Wechselstabtechnik ein anterolaterales Optikportal angelegt.

Am Schlüsselbein wird etwa $3,5 \mathrm{~cm}$ medial des ACG eine $2-3 \mathrm{~cm}$ lange Inzision vorgenommen und die Klavikula in a.-p. Ausdehnung dargestellt. Die Korakoidbasis wird in mediolateraler Ausdehnung präpariert ( $\bullet$ Abb. 2d), und der Teller des Zielbügels wird über das anteroinferiore Portal unter visueller Kontrolle mittig basisnah am Korakoid platziert. Nach Reposition über den pneumatischen Armhalter wird bildwandlergestützt die Position für die Bohrung festgelegt (• Abb. 2a) und der Zieldraht vorgebohrt. Dabei wird auf $4 \mathrm{~mm}$ überbohrt (- Abb. 2b) und das Implantat über einen Shuttledraht eingezogen. Die Positionierung erfolgt unter Zuhilfenahme einer Fasszange (- Abb. 2e). Der subkorakoidale Fixationsbutton liegt idealerweise basisnah unter dem Korakoid (- Abb. 2f, - Abb.3). Es folgt die Reposition mit einem Kugelspieß durch Druck der lateralen Klavikula nach kaudal. Abschließend werden die FiberWire ${ }^{\text {Tw }}$-Fäden über dem klavikularen Fixationsbutton verknotet.
Bei horizontaler Instabilität erfolgt eine Augmentation durch eine transklavikulare, transkorakoidale FiberTa$\mathrm{pe}^{\mathrm{Tm}}$-Cerclage. Hierzu wird die Klavikula mittels eines Zieldrahts über das anteroinferiore Portal horizontal durchbohrt und anschließend über einen kanülierten Bohrer auf 2,7 mm aufgebohrt. Über das anterolaterale Portal wird unter Bildverstärkerkontrolle analog am Akromion von laterokaudal nach mediokranial gebohrt (- Abb. 2c). Über Durchzugsdrähte wird die Cerclage durch die Klavikula gezogen, anschließend wird ein Ende durch die transakromiale Bohrung geführt, mit einer Fasszange subkutan nach medial zurückgeholt und dort verknotet. Es folgt der schichtweise Wundverschluss mit sorgfältiger Fasziennaht. 


\section{Schulterverletzungen}
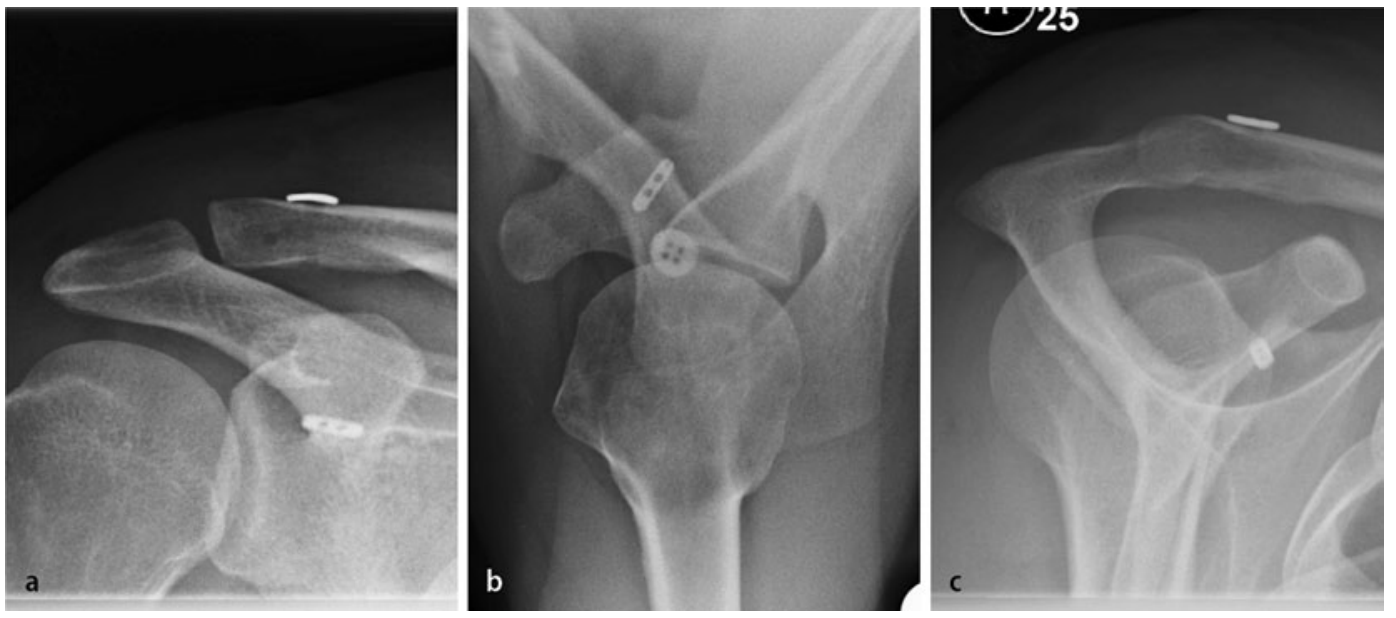

Abb. 34 Postoperatives Röntgenergebnis 6 Monate nach Einzel-Tight-RopeStabilisierung mit horizontaler FiberTape ${ }^{\mathrm{TM}}$-Augmentation, a ACG-Zielaufnahme mit Belastung, b Positionierung der Fixationsbuttons mittig in der Klavikula und der Korakoidbasis, cY-Aufnahme: Platzierung des Buttons basisnah am Korakoid, ACG Akromioklavikulargelenk
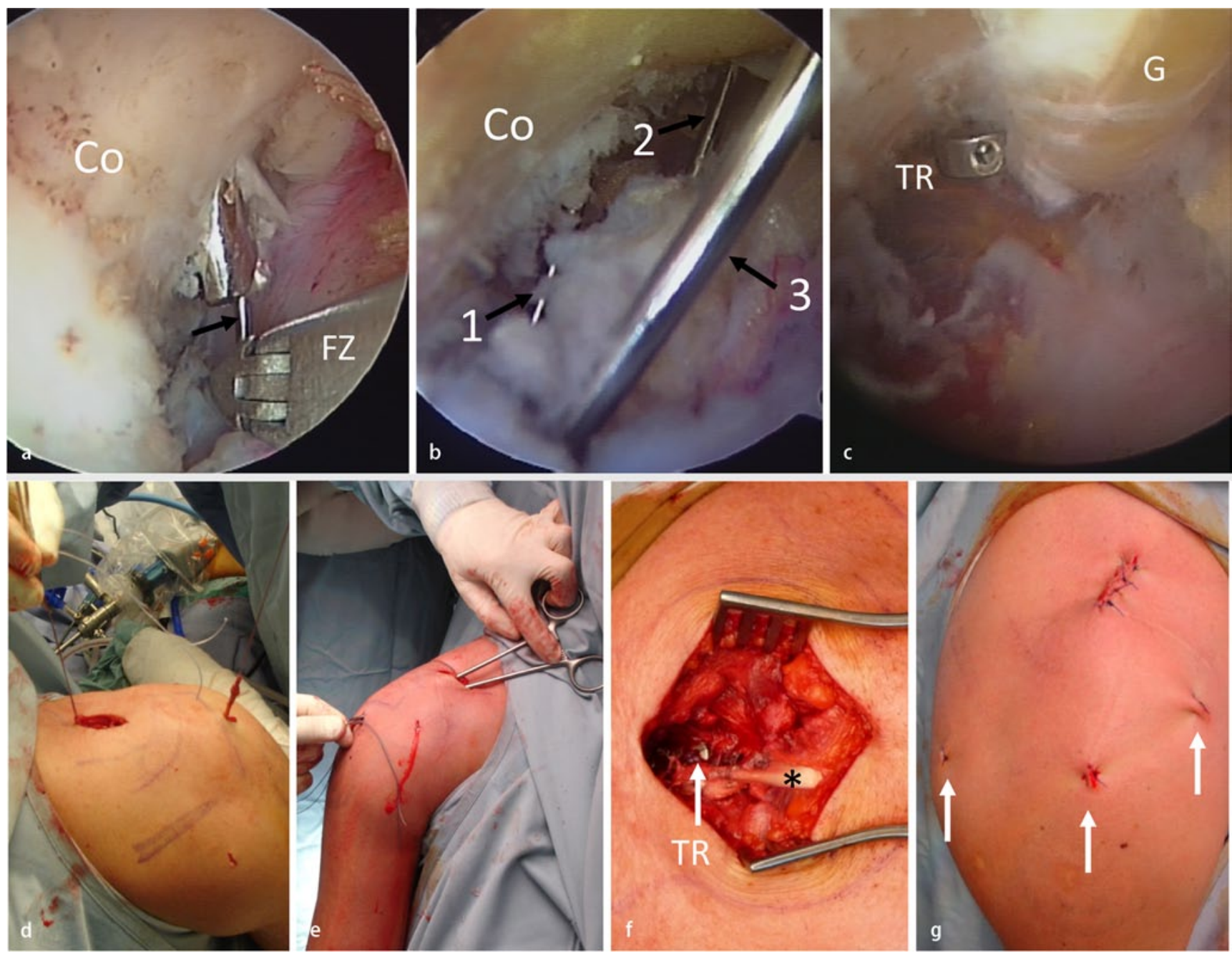

Abb. 4 \ Operationstechnik bei chronischer ACG-Instabilität, a Platzierung der transklavikularen-transkorakoidalen Bohrkanäle, Co Korakoidbasis, Fz Fasszange, schwarzer Pfeil Nitinoldurchzugsdraht, b Lage der Durchzugsdrähte 1 Draht für den Tight-Rope, 2 Draht für die Grazilissehne, 3 Position des 3. Drahtes transklavikular und lateral der Korakoidbasis, c nach Platzierung des Tight-Rope (TR) und Einziehen der Grazilissehne (G), d,e Ausziehen des langen Sehnenendes über das anterolaterale Portal (d) und Rückführung nach medial (e), f Vernähen der Sehnenenden (Sternchen), $\mathbf{g}$ nach Wundverschluss, weiße Pfeile Arthroskopieportale, weitere Erläuterungen s. Text. (Nach [16]) 


\section{Chronische ACG-Instabilität}

Etwa 1/5 aller operativ oder konservativ behandelten ACG-Instabilitäten werden im Verlauf chronisch-symptomatisch [28]. Gründe dafür sind eine verbliebene vertikale Instabilität nach Versagen einer operativen oder konservativen Therapie, eine verbliebene oder übersehene horizontale Instabilität mit Unterschätzung des Verletzungsausmaßes oder die Entwicklung einer posttraumatische Arthrose [28].

Nach einem Zeitintervall von $>3$ Wochen nach einem Trauma scheint die regenerative Kapazität der CC- und AC-Bänder zur Bildung einer stabilen Narbe nicht mehr ausreichend, sodass eine alleinige Stabilisierung mit einem Implantat nicht mehr Erfolg versprechend ist und biologische Ersatzverfahren erforderlich werden.

Auch in diesem Bereich konnten sich neben den konventionellen Operationstechniken wie dem extraanatomischen Weaver-Dunn-Verfahren oder dem anatomischen Ansatz einer autologen Sehnenersatzplastik der CCL im Lauf der letzten Jahre zunehmend arthroskopisch gestützte Techniken etablieren $[3,25]$.

Die autologe CCL-Plastik ist der Transposition des korakoakromialen Bandes nach Weaver-Dunn in ihren Modifikation in biomechanischer Hinsicht überlegen [30]. In einer prospektiven Studie konnten Tauber et al. [28] mit einer autologen Semitendinosusplastik der CCL signifikant bessere Resultate als mit einer modifizierten Weaver-Dunn-Technik erzielen.

\section{Arthroskopisch assistierte Operationstechnik bei chronischer Instabilität}

Im eigenen Vorgehen erfolgt eine arthroskopisch gestützte CCL-Plastik mit einer Grazilissehne sowie einer Retention durch ein Tight-Rope-Implantat gemäß der von Scheibel et al. [25] veröffentlichten Technik. Diese wird um eine intraossäre transakromiale Grazilisschlinge zur zusätzlichen horizontalen Augmentation erweitert, um insbesondere das klinisch relevante Problem der horizontalen Instabilität gesondert zu adressieren $[13,16]$.

Die Portale für die Arthroskopie sind identisch wie bei der Einzel-Tight-Ro-
pe-Technik, die Inzision über der lateralen Klavikula sollte jedoch länger sein (etwa 3-4 cm). Die Grazilissehne als autologes Sehnentransplantat wird ipsilateral entnommen, an den Enden armiert und so lang wie möglich belassen (mindestens $20 \mathrm{~cm}$ ). Die transklavikularen-transkorakoidalen Bohrkanäle werden über das Zielinstrument unter visueller sowie Bildverstärkerkontrolle platziert (- Abb. 4a). Sie dienen zur Einführung des Drahtes für den Tight-Rope und die Grazilissehne, der 3. Draht wird transklavikular und lateral an der Korakoidbasis vorbei geführt (- Abb. 4b). Der Tight-Rope wird analog der Technik bei akuter Instabilität an vorgesehener Stelle platziert. Die Grazilissehne wird mit Hilfe der Durchzugsdrähte durch die mediale Bohrung an der Klavikula und dem Korakoid transossär ein- und U-förmig lateral am Korakoid vorbei zurückgezogen ( $\bullet$ Abb. 4c). Die Sehne wird in den klavikularen Bohrungen durch Tenodeseschrauben gesichert. Die Platzierung der transakromialen Bohrung erfolgt analog der Technik für die beschriebene FiberTape ${ }^{\mathrm{Tm}}$-Augmentation bei akuter Instabilität. Das lange Ende der Sehne wird durch das Akromion über das anterolaterale Portal ausgezogen (- Abb.4d) und nach medial zurückgeführt (- Abb. 4e). Die Sehnenenden werden miteinander vernäht ( $\bullet$ Abb. 4f). Es folgt der sorgfältige Verschluss der Faszie sowie der Wunde (• Abb.4g).

\section{Nachbehandlung}

Nach einer arthroskopisch gestützten Stabilisierung des ACG ist eine 6-wöchige Nachbehandlung in einer SchulterArm-Schlinge indiziert. Wichtig ist, dass die Orthese das Eigengewicht des Arms möglichst reduziert. Während der ersten 2 Wochen ist im eigenen Vorgehen lediglich eine passive Beübung aus der Orthese heraus bis zu einer Anteversion und Abduktion bis $30^{\circ}$ erlaubt. In der 3 . und 4. Woche ist eine aktiv-assistive Bewegung jeweils bis $45^{\circ}$ erlaubt, welche in den Wochen 5 und 6 auf $60^{\circ}$ gesteigert wird. Ab Woche 7 wird die Beweglichkeit im Schultergelenk im schmerzfreien Rahmen vollständig freigegeben. Beübung gegen Widerstand ist ab der 12 . Woche möglich, sollte jedoch bei autologen Sehnenplasti- ken zurückhaltender erfolgen als bei der Versorgung akuter Instabilitäten.

\section{Fazit für die Praxis}

- Eine adäquate Röntgendiagnostik ist zur Erfassung der vertikalen und horizontalen Instabilität wichtig.

- Verbliebene horizontale Instabilitäten gelten bei der Rockwood-III-Verletzung als Prädiktor für ein schlechtes Outcome.

- Alle Komponenten der Instabilität sollten in das therapeutische Konzept integriert werden.

- Mit arthroskopisch gestützten Techniken können sehr gute Resultate erzielt werden.

- Intraartikuläre Begleitpathologien können bei der arthroskopisch gestützten Technik detektiert und einzeitig mit adressiert werden.

- Bei chronischen Instabilitäten werden biologische Ersatzverfahren wie eine CCL-Plastik mit autologer Sehne erforderlich.

\section{Korrespondenzadresse}

Prof. Dr. H. Lill

Abteilung für Unfall- und Wiederherstellungschirurgie, Diakoniekrankenhaus Friederikenstift gGmbH Hannover, Abteilung des Berufsgenossenschaftlichen Unfallkrankenhauses Hamburg, Humboldtstraße 5, 30169 Hannover helmut.lill@ddh-gruppe.de

Interessenkonflikt. Der korrespondierende Autor gibt für sich und seine Koautoren an, dass kein Interessenkonflikt besteht.

The supplement containing this article is not sponsored by industry.

\section{Literatur}

1. Alexander OM (1949) Dislocation of the acromioclavicular joint. Radiography 15(179):260

2. Beitzel K, Sablan N, Chowaniec DM, Obopilwe E, Cote MP, Arciero RA, Mazzocca AD (2012) Translation sequential resection of the distal clavicle and its effects on horizontal acromioclavicular joint. Am J Sports Med 40:681-685

3. Boileau P, Old J, Gastaud O, Brassart N, Roussanne Y (2010) All-arthroscopic Weaver-Dunn-Chuinard procedure with double-button fixation for chronic acromioclavicular joint dislocation. Arthroscopy $26(2): 149-160$ 
4. Ceccarelli E, Bondi R, Alviti F, Garofalo R, Miulli F, Padua R (2008) Treatment of acute grade III acromioclavicular dislocation: a lack of evidence. J Orthop Trauma 9(2):105-108

5. EIMaraghy AW, Devereaux MW, Ravichandiran K, Agur AM (2010) Subacromial morphometric assessment of the clavicle hook plate. Injury 41(6):613-619

6. Dias JJ, Gregg PJ (1991) Acromioclavicular joint injuries in sport. Recommendations for treatment. Sports Med 11:125-132

7. Fukuda K, Craig E, An KN, Cofield RH, Chao EY (1986) Biomechanical study of the ligamentous system of the acromioclavicular joint. J Bone Joint Surg Am 68:434-440

8. Gerhardt C, Kraus N, Greiner S, Scheibel M (2011) Arthroskopische Stabilisierung der akuten Schultereckgelenksprengung. Orthopade 40(1):61-69

9. Gumina S, Carbone S, Postacchini F (2009) Scapular dyskinesis and sick scapula syndrome in patients with chronic type III acromioclavicular dislocation. Arthroscopy 25(1):40-45

10. Gstettner C, Tauber M, Hitzl W, Resch H (2008) Rockwood type III acromio-clavicular dislocation: surgical versus conservative treatment. J Shoulder Elbow Surg 17(2):220-225

11. Hedtmann A, Fett H, Ludwig J (1998) Management of old neglected posttraumatic acromioclavicular joint instability and arthrosis. Orthopade 27(8):556-566

12. Jensen $G$, Katthagen JC, Alvarado L, Voigt C, Lill H (2011) Ist die arthroskopisch gestützte DoppelTight-Rope ${ }^{\mathrm{T} M}$-Technik bei akuter ACG-Sprengung der Hakenplatte überlegen? 18. Jahreskongress der deutschen Gesellschaft für Schulter- und Ellenbogenchirurgie, Stuttgart

13. Jensen G, Katthagen JC, Alvarado L, Voigt C, Lill H (2011) Die arthroskopisch gestützte Stabilisierung chronischer ACG-Instabilitäten in Graft-Rope ${ }^{\mathrm{TM}}$ Technik mit vertikaler und horizontaler Sehnenaugmentation. 18. Jahreskongress der deutschen Gesellschaft für Schulter- und Ellenbogenchirurgie, Stuttgart

14. Kienast B, Thietje R, Queitsch C, Gille J, Schulz AP, Meiners J (2011) Mid-term results after operative treatment of Rockwood grade III-V acromioclavicular joint dislocations with an AC-hook-plate. Eur J Med Res 16(2):52-56

15. Klimkiewicz JJ, Williams GR, Sher JS, Karduna A, Des Jardins J, lannotti JP (1999) The acromioclavicular capsule as a restraint to posterior translation of the clavicle: a biomechanical analysis. J Shoulder Elbow Surg 8(2):119-124

16. Kraus N, Gerhardt C, Greiner S, Scheibel M (2010) Arthroskopische Behandlungsmöglichkeiten chronischer Schultereckgelenksinstabilitäten. Arthroskopie 23:293-303

17. Mazzocca AD, Arciero RA, Bicos J (2007) Evaluation and treatment of acromioclavicular joint injuries. Am J Sports Med 35(2):316-29

18. Mouhsine E, Garofalo R, Crevoisier X, Farron A (2003) Grade I and II acromioclavicular dislocations: results of conservative treatment. J Shoulder Elbow Surg 12(6):599-602

19. NN (2010) Meeting des Komittees Trauma der Gesellschaft für Arthroskopie und Gelenkchirurgie. Deutscher Kongress für Orthopädie und Unfallchirurgie, Berlin

20. Patzer T, Clauss C, Kühne CA, Ziring E, Efe T, Ruchholtz S, Mann D (2012) Arthroscopically assisted reduction of acute acromioclavicular joint separations: comparison of clinical and radiological results of single versus double TightRope ${ }^{\mathrm{TM}}$ technique. Unfallchirurg Jan 20. [Epub ahead of print]
21. Pauly S, Gerhardt C, Haas NP, Scheibel M (2009) Prevalence of concomitant intraarticular lesions in patients treated operatively for high-grade acromioclavicular joint separations. Knee Surg Sports Traumatol Arthrosc 17(5):513-517

22. Petersson C (1983) Degeneration of the acromioclavicular joint: a morphological study. Acta Orthop Scand 54:434-438

23. Rockwood C (1984) Injuries in the acromioclavicular joint - sublocations and dislocations about the shoulder. In: Rockwood C Jr, Green D (Hrsg) Fractures in adults. Lippincott, Philadelphia, S 860-910

24. Rolf O, Hann von Weyhern A, Ewers A, Boehm TD, Gohlke F (2008) Acromioclavicular dislocation Rockwood III-V: results of early versus delayed surgical treatment. Arch Orthop Trauma Surg 128(10):1153-1157

25. Scheibel M, Ifesanya A, Pauly S, Haas NP (2008) Arthroscopically assisted coracoclavicular ligament reconstruction for chronic acromioclavicular joint instability. Arch Orthop Trauma Surg 128(11):1327-1333

26. Scheibel M, Dröschel S, Gerhardt C, Kraus N (2011) Arthroscopically assisted stabilization of acute high-grade acromioclavicular joint separations. Am J Sports Med 39(7):1507-1516

27. Smith TO, Chester R, Pearse EO, Hing CB (2011) Operative versus non-operative management following Rockwood grade III acromioclavicular separation: a meta-analysis of the current evidence base. J Orthop Trauma 12(1):19-27

28. Tauber M, Gordon K, Koller H, Fox M, Resch H (2009) Semitendinosus tendon graft versus a modified Weaver-Dunn procedure for acromioclavicular joint reconstruction in chronic cases: a prospective comparative study. Am J Sports Med 37(1):181-190

29. Tauber M, Koller H, Hitzl W, Resch H (2010) Dynamic radiologic evaluation of horizontal instability in acute acromioclavicular joint dislocations. Am J Sports Med 38(6):1188-1195

30. Thomas K, Litsky A, Jones G, Bishop JY (2011) Biomechanical comparison of coracoclavicular reconstructive techniques. Am J Sports Med 39(4):804-810

31. Tischer T, Salzmann GM, El-Azab H, Vogt S, Imhoff $A B(2009)$ Incidence of associated injuries with acute acromioclavicular joint dislocations types III through V. Am J Sports Med 37(1):136-139

32. Tossy JD, Mead NC, Sigmond HM (1963) Acromioclavicular separations: useful and practical classification for treatment. Clin Orthop Relat Res 28:111-119 